\title{
Superação de dormência de sementes como estratégia para restauração florestal de pastagem tropical
}

\author{
Silvia Rahe Pereira(1), Valdemir Antônio Laura(2) e Andréa Lúcia Teixeira de Souza(3)
}

\begin{abstract}
(1)Universidade Federal de São Carlos (UFSCAR), Programa de Pós-Graduação em Ecologia e Recursos Naturais, Rodovia Washington Luiz, Km 235, Caixa Postal 676, CEP 13565-905 São Carlos, SP. E-mail: silviarahe@gmail.com (2)Embrapa Gado de Corte, BR-262, Km 4, Caixa Postal 154, CEP 79002-970 Campo Grande, MS. E-mail: valdemir.laura@embrapa.br ${ }^{(3)}$ UFSCAR, Departamento de Ciências Ambientais. E-mail: altdesouza@gmail.com
\end{abstract}

\begin{abstract}
Resumo - O objetivo deste trabalho foi avaliar a influência de tratamentos de superação da dormência de sementes sobre a taxa e o tempo de emergência de espécies arbóreas de Fabaceae, e sobre seu estabelecimento, quando reintroduzidas em pastagem, no Cerrado. Foram realizados experimentos em casa de vegetação e em campo (pastagem de Urochloa brizantha), com as espécies Mimosa caesalpiniifolia, Peltophorum dubium, Pterogyne nitens, Dimorphandra mollis, Copaifera langsdorffi e Hymenaea stigonocarpa, submetidas ou não aos seguintes tratamentos de superação de dormência: D. mollis e H. stigonocarpa, escarificação mecânica; C. langsdorffii e $M$. caesalpiniifolia, escarificação química com $\mathrm{H}_{2} \mathrm{SO}_{4} ;$ P. nitens, punção do tegumento; e P. dubium, imersão em água a $80^{\circ} \mathrm{C}$. O estabelecimento em campo foi monitorado por 21 meses. A superação de dormência promoveu, em casa de vegetação, maior emergência de quatro das seis espécies avaliadas, e, no campo, de cinco espécies. Além disso, reduziu o tempo de emergência das espécies e aumentou o recrutamento de M. caesalpiniifolia, P. nitens, D. mollis e H. stigonocarpa, aos 127 dias, e de H. stigonocarpa aos 659 dias. Os tratamentos de superação de dormência podem aumentar a eficiência da semeadura direta, em projetos de restauração de áreas degradadas no Cerrado, ao promover maior emergência das sementes e o estabelecimento das plantas.
\end{abstract}

Termos para indexação: fabáceas arbóreas, recrutamento, restauração de área degradada, semeadura direta, tempo de emergência.

\section{Seed dormancy overcoming as a strategy for forest restoration in tropical pasture}

\begin{abstract}
The objective of this work was to evaluate the influence of seed dormancy overcoming treatments on the rate and time of emergence of Fabaceae tree species, and on their recruitment when reintroduced in a tropical pasture, in the Brazilian Cerrado. Experiments were carried out in greenhouse and field (Urochloa brizantha pasture), with the species Mimosa caesalpiniifolia, Peltophorum dubium, Pterogyne nitens, Dimorphandra mollis, Copaifera langsdorffii and Hymenaea stigonocarpa, subjected or not to the following treatments of dormancy break: D. mollis and H. stigonocarpa, mechanical scarification; C. langsdorffi and M. caesapiniifolia, chemical scarification with $\mathrm{H}_{2} \mathrm{SO}_{4} ;$; . nitens, tegument puncture; and P. dubium, immersion in water at $80^{\circ} \mathrm{C}$. Recruitment of plants in the field were followed up for 21 months. Dormancy overcoming promoted greater emergency in four out of the six tested species, in the greenhouse, and in five species in the field. Furthermore, it reduced the emergency timing of the species and increased the recruitment of M. caesalpiniifolia, P. nitens, D. mollis e H. stigonocarpa, at 127 days, and of H. stigonocarpa at 659 days. Seed dormancy overcoming treatments can increase the efficiency of direct seeding in restoration projects of degraded areas in the Cerrado, promoting greater seed emergency and the recruitment of plants.
\end{abstract}

Index terms: Fabaceae trees, recruitment, degraded areas restoration, direct seeding, timing of emergence.

\section{Introdução}

Nas últimas décadas, os ecossistemas tropicais foram intensamente convertidos em pastagens (Cheung et al., 2010) e, recentemente, a necessidade de restauração dessas áreas para recuperação de funções e serviços dos ecossistemas tem aumentado (Florentine \&
Westbrooke, 2004; Viglizzo \& Frank, 2006). Estudos recentes têm mostrado a eficácia da utilização de semeadura direta de espécies arbóreas em projetos de restauração, como técnica de menor custo financeiro e de mais fácil operacionalização que plantio de mudas (Camargo et al., 2002; Woods \& Elliott, 2004; Sovu et al., 2010). Segundo Mattei \& Rosenthal (2002),

Pesq. agropec. bras., Brasília, v.48, n.2, p.148-156, fev. 2013

DOI: 10.1590/S0100-204X2013000200004 
há poucos exemplos de implantação de florestas por semeadura direta na América Latina, e Florentine \& Westbrooke (2004) ressaltaram a necessidade de experimentos que avaliem a eficiência dessa técnica em áreas de pastagens abandonadas.

O sucesso da utilização da semeadura direta depende da escolha de espécies adaptadas ou tolerantes às condições ambientais locais (Sovu et al., 2010). Nesse sentido, as fabáceas despertam grande interesse, já que são capazes, em sua grande maioria, de fixar nitrogênio da atmosfera (Siddique et al., 2008) e contribuem para a recuperação do solo, o que propicia condições mais favoráveis ao estabelecimento de outras espécies (Franco et al., 1992). Contudo, muitas das sementes dessas espécies apresentam dormência física (Andrade et al., 1997), com tegumento total ou parcialmente impermeável à água.

A dormência de sementes é uma das principais estratégias utilizadas por espécies vegetais para aumentar suas taxas de sobrevivência e o estabelecimento de plantas jovens (McIvor \& Howden, 2000), pois possibilita que a germinação ocorra apenas quando é mais provável que as condições para o estabelecimento das plântulas sejam mais adequadas (Finch-Savage \& Leubner-Metzger, 2006). Assim, a redução do tempo de emergência de plântulas, induzida pela superação artificial da dormência das sementes, poderia resultar em menor recrutamento de plantas jovens. No entanto, em áreas com estações seca e chuvosa bem definidas, como é o caso do Cerrado, um maior controle do tempo de emergência, por meio da superação da dormência, poderia diminuir as taxas de mortalidade por dessecação e aumentar o tempo que as plântulas emergidas teriam durante a estação de crescimento, antes do início de uma nova estação seca (Woods \& Elliott, 2004; Schmidt, 2008). Além disso, a redução do tempo de emergência pode também diminuir o tempo de exposição das sementes a fungos e outros patógenos (Dalling et al., 2011) e a predadores vertebrados e invertebrados (Doust et al., 2008), que são fatores pós-dispersão limitantes ao estabelecimento de espécies de árvores em áreas de pastagens.

Embora procedimentos pré-germinativos para superação de dormência já tenham sido usados em estudos que avaliaram a semeadura direta como técnica de restauração (Camargo et al., 2002; Doust et al., 2008; Bonilla-Moheno \& Holl, 2010), as informações sobre possíveis efeitos do uso deste procedimento no sucesso de germinação e na sobrevivência das plântulas ainda são escassas, especialmente em condições de campo.

Especificamente, o experimento avaliou se o tratamento de superação de dormência pode aumentar as taxas de emergência em casa de vegetação e em campo, reduzir efetivamente o tempo de emergência em condições de campo e, também, se influencia o estabelecimento de plantas jovens nos primeiros 21 meses após a semeadura.

O objetivo deste trabalho foi avaliar a influência de tratamentos de superação da dormência de sementes sobre a taxa e o tempo de emergência de espécies de Fabaceae, e sobre seu estabelecimento, quando reintroduzidas em pastagem, no Cerrado.

\section{Material e Métodos}

O estudo foi realizado em uma área experimental de pastagem composta de Urochloa brizantha (Hoschst. Ex A. Rich), da Embrapa Gado de Corte, em Campo Grande, MS (20²5'27"S, 5441'16"W, a $566 \mathrm{~m}$ de altitude). O clima da região, segundo a classificação de Köppen, é do tipo Aw, tropical úmido, com estação chuvosa no verão e seca no inverno. O solo da área é do tipo Latossolo Vermelho distroférrico (Melotto, 2008). Originalmente, a área era ocupada pela formação de cerrado senso estrito. Quinze anos antes da implantação do experimento, a vegetação original foi suprimida e, então, a gramínea exótica $U$. brizantha foi implantada, para formação de pastagem.

Foram realizados experimentos em casa de vegetação e em campo, com seis espécies da família Fabaceae, para avaliar o efeito da superação de dormência de sementes sobre as taxas e o tempo de emergência, bem como sobre o estabelecimento de plantasjovens no campo. O estabelecimento das plantas foi monitorado por 21 meses. Um lote de 800 sementes de cada espécie foi usado nos experimentos, tendose estimado a massa média das sementes a partir dos valores da massa de 1.000 sementes (Regras para análise de sementes, 2009). As espécies utilizadas, com suas respectivas massas médias de sementes, foram: Mimosa caesalpiniifolia Benth., 0,03g; Peltophorum dubium (Spreng.) Taub., 0,05 g; Pterogyne nitens Tul., 0,11 g; Dimorphandra mollis Benth., 0,24 g; Copaifera langsdorffii Desf., 0,48 g; 
e Hymenaea stigonocarpa Mart. Ex Hayne, 3,42 g. As sementes de $H$. stigonocarpa, P. nitens, $P$. dubium e M. caesalpiniifolia foram coletadas em fragmentos de cerrado de Campo Grande, Mato Grosso do Sul, e as de $C$. langsdorffii e $D$. mollis foram coletadas em fragmentos de cerrado de São Carlos, São Paulo.

A superação de dormência das sementes de $D$. mollis (Salomão et al., 2003) e H. stigonocarpa (Pereira et al., 2011) foi realizada por meio de escarificação mecânica. Sementes de $C$. langsdorffii e $M$. caesalpiniifolia foram submetidas à escarificação química com $\mathrm{H}_{2} \mathrm{SO}_{4}$, por 5 min (Salomão et al., 2003) e 7 min (Martins et al., 1992), respectivamente. Em P. nitens, utilizou-se a punção do tegumento (Nassif \& Perez, 1997) e, em P. dubium, imersão em água a $80^{\circ} \mathrm{C}$ por 1 min (Oliveira et al., 2008).

O experimento de campo foi conduzido em blocos ao acaso, em arranjo fatorial $6 \times 2$. Foram delimitados quatro blocos, com todas as combinações de tratamentos. Dentro de cada bloco, foram marcadas seis parcelas contíguas de $10 \times 1 \mathrm{~m}$, tendo-se designado uma para cada espécie. A posição das espécies dentro dos blocos foi aleatória. Nas parcelas, foram dispostos 20 conjuntos de cinco sementes, dos quais dez conjuntos com superação de dormência e 10 sem superação, em posições alternadas dentro das parcelas, o que totalizou 400 sementes de cada espécie. As sementes foram enterradas a uma profundidade de aproximadamente três vezes sua espessura (menor dimensão da semente), para diminuir o efeito de predação (Woods \& Elliott, 2004). As áreas em que os blocos foram demarcados receberam manejo prévio de remoção de gramíneas, para reduzir o efeito de competição interespecífica. A remoção de gramíneas foi realizada com aplicação do herbicida glifosato, não tendo sido realizados controles posteriores.

A semeadura foi realizada em 04/02/2009, e o experimento foi monitorado quinzenalmente, nas primeiras 10 semanas, mensalmente, até que fossem completadas 58 semanas, e bimestralmente, até que fossem completados 21 meses. O número de sementes emergidas, o tempo de emergência e o recrutamento, expresso pela relação entre o número de indivíduos vivos e o número de sementes semeadas, foram considerados como variáveis-respostas. Considerouse como emergidas as plântulas que apresentaram expansão completa dos eófilos. Todos os indivíduos foram etiquetados.
Um segundo experimento foi realizado em casa de vegetação, para a análise, em condições controladas, da emergência de plântulas das seis espécies. Para cada espécie, foram utilizadas quatro repetições de 50 sementes com superação de dormência e quatro repetições de 50 sementes sem superação de dormência. As sementes foram semeadas em bandejas de isopor, com substrato Plantmax, mantidas em casa de vegetação e umedecidas dez vezes ao dia durante 10 min, com uso de nebulizadores com vazão de $7 \mathrm{~L}$ por hora. O número de sementes emergidas durante 24 meses foi registrado por meio de contagens semanais, durante o primeiro mês do experimento, quinzenais, nos três meses subsequentes, mensais, até que fosse completado um ano de experimento, e bimestrais, até o final do experimento. $\mathrm{O}$ número de plantas emergidas e o tempo de emergência (dias) foram considerados como variáveis respostas.

Ainfluência do tratamento de superação de dormência na probabilidade de emergência de indivíduos, tanto no experimento de campo, como no experimento em casa de vegetação, foi avaliada por meio da análise de regressão logística, conduzida para as seis espécies separadamente. $\mathrm{O}$ modelo incluiu a resposta binária (emergência ou não de cada semente individual) como variável resposta e o tratamento de superação de dormência como variável explicativa. $\mathrm{O}$ valor de "razão de chances" (odds ratio) indica quantas vezes a probabilidade de emergência de plantas de sementes, no tratamento CS (com superação), é maior do que a probabilidade de emergência de plantas de sementes no tratamento (SS) considerado como referência (Jaccard, 2001). Assim, valores de "razão de chances" maiores do que 1 representam efeito positivo do tratamento CS em comparação ao tratamento SS.

O efeito do tratamento de superação de dormência, no tempo de emergência, em ambos os experimentos, foi avaliado por meio da análise de tempo de evento ("failure-time analysis") (Fox 2001). Para cada nível de tratamento (SS e CS), esta análise ajustou uma curva que descreveu a relação entre o número de eventos de emergência ou não emergência (variável dependente), ao longo do tempo (variável explicativa). Esta abordagem estatística compara os valores preditos de tempo, em que $50 \%$ das sementes tenham emergido, entre os dois níveis de tratamento.

A influência da superação de dormência sobre o número de indivíduos recrutados das diferentes 
espécies, ao longo do tempo, no experimento de campo, foi avaliada pela análise de variância de medidas repetidas, para cada espécie separadamente. Esta análise permitiu avaliar o efeito do tratamento de superação de dormência sobre o recrutamento, ao longo do tempo. Três tempos foram usados: 127 dias, quando mais de $95 \%$ das plântulas do tratamento "com superação de dormência" haviam emergido; 320 dias, quando mais de $95 \%$ de todas as sementes, com e sem superação de dormência, haviam emergido, e ao final do experimento, após 659 dias. Quando a interação entre o tratamento e o tempo foi significativa, procedeu-se à análise de variância, para se avaliar como o efeito do tratamento variou entre os três tempos. Os resíduos foram conferidos graficamente, para verificação da existência de heterogeneidade de variâncias ou presença de valores atípicos, que poderiam invalidar a análise. Os dados referentes ao número de indivíduos recrutados foram transformados em logaritmo natural, para homogeneização das variâncias. Considerou-se a probabilidade de $5 \%$, em todas as análises descritas.

\section{Resultados e Discussão}

O efeito da superação de dormência na probabilidade de emergência de plântulas variou entre espécies, em casa de vegetação e em campo (Tabela 1). Em casa de vegetação, a probabilidade de emergência de $P$. nitens e C. langsdorffii não diferiu entre os tratamentos (Tabela 1, Figura 1). No entanto, a superação da dormência resultou em aumento da probabilidade de emergência de $M$. caesalpiniifolia, $P$. dubium,
D. mollis, e H. stigonocarpa. Em campo, a superação de dormência de sementes diminuiu a taxa de emergência de plântulas de C. langsdorffii, mas levou ao aumento da probabilidade de emergência das demais espécies. A utilização do tratamento pré-germinativo resultou também em redução significativa do tempo de emergência, em todas as espécies avaliadas, tanto em casa de vegetação como em campo (Tabela 2, Figura 1).

Todas as espécies avaliadas apresentam dormência física das sementes, com tegumento impermeável à água até o início do processo de germinação. Esta propriedade é considerada como uma estratégia de defesa à penetração de patógenos (Dalling et al., 2011). Segundo os autores, esta estratégia é comum em espécies de florestas tropicais, em que as condições úmidas e quentes favorecem o crescimento de fungos e bactérias. Contudo, o experimento de campo, no presente estudo, foi realizado em área de pastagem, cujas condições ambientais diferem muito das condições do ecossistema original, com menor umidade nos microssítios de germinação (Nepstad et al., 1996), o que pode ter diminuído as chances de mortalidade por patógenos, nas sementes que tiveram a dormência superada. No entanto, as condições de baixa umidade nas áreas de pastagem, no Cerrado, restringem muito a época favorável para a emergência das sementes. Nestas condições, quando não se utiliza a superação de dormência, as sementes demoram mais tempo para iniciar o processo de germinação, o que pode ocorrer quando a disponibilidade de água já encontra-se muito reduzida. Sem a superação da dormência, algumas

Tabela 1. Resultado das análises de regressão logística, do efeito do tratamento de superação de dormência sobre a probabilidade de emergência de plântulas, em casa de vegetação e no campo.

\begin{tabular}{|c|c|c|c|c|}
\hline \multirow[t]{2}{*}{ Espécie } & \multicolumn{2}{|c|}{ Casa de vegetação } & \multicolumn{2}{|l|}{ Campo } \\
\hline & “Razão de chances" \pm erro-padrão ${ }^{(1)}$ & Modelo geral $\chi^{2}$ & "Razão de chances" \pm erro-padrão & Modelo geral $\chi^{2}$ \\
\hline Mimosa caesalpiniifolia & $11,074 \pm 2,926$ & $105,704 * * *$ & $8,483 \pm 2,113$ & $88,632 * * *$ \\
\hline Peltophorum dubium & $1,701 \pm 0,361$ & $6,353 *$ & $1,749 \pm 0,358$ & $7,520 * *$ \\
\hline Pterogyne nitens & $1,272 \pm 0,255$ & $1,441^{\mathrm{ns}}$ & $2,236 \pm 0,481$ & $14,363 * * *$ \\
\hline Dimorphandra mollis & $9,692 \pm 2,372$ & $103,707 * * *$ & $2,889 \pm 0,683$ & $21,345^{* * *}$ \\
\hline Copaifera langsdorffii & $1,741 \pm 0,661$ & $2,19^{\mathrm{ns}}$ & $0,532 \pm 0,114$ & $8,781 * *$ \\
\hline Hymenaea stigonocarpa & $56,435 \pm 17,529$ & $265,001 * * *$ & $5,130 \pm 1,357$ & $44,399 * * *$ \\
\hline
\end{tabular}

${ }^{(1)} \mathrm{O}$ termo "razão de chances" indica quantas vezes a probabilidade de emergência de plantas, originadas de sementes submetidas ao tratamento com superação de dormência, é maior do que a probabilidade de emergência de plantas de sementes sem superação de dormência. ${ }^{\text {ns }}$ Não significativo. ${ }^{*}, * * e^{* * *}$ Significativo, pela regressão logística, a 5,1 e $0,1 \%$ de probabilidade, respectivamente. 

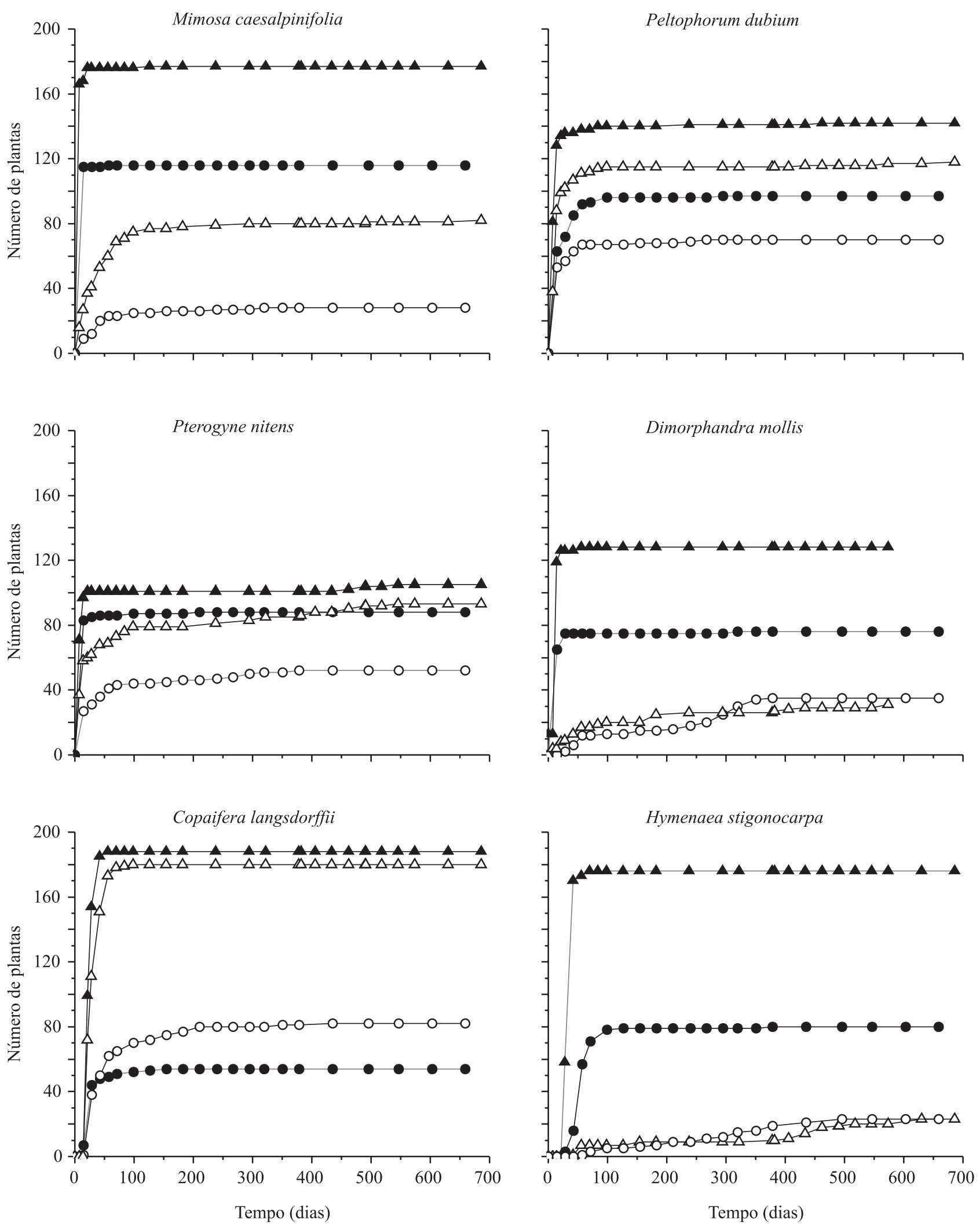

Figura 1. Germinação cumulativa das espécies avaliadas em casa de vegetação (triângulos) e em campo (círculos), submetidas aos tratamentos com superação de dormência (símbolos cheios) e sem superação de dormência (símbolos vazios). 
sementes - na maioria das espécies - levaram até junho para emergir, época em que a precipitação havia caído para níveis muito baixos, o que pode ter levado à falha do processo de germinação e morte das sementes. Além disso, um maior tempo de emergência leva à exposição prolongada a inimigos naturais no solo, o que aumenta as chances de mortalidade dos indivíduos ainda em fase de semente. Assim, o uso da superação de dormência aumentou a probabilidade de emergência de todas as espécies avaliadas, com exceção de C. langsdorffii em campo.

Os resultados de emergência de $C$. langsdorffii em campo não eram esperados, uma vez que outros autores indicam a utilização da superação de dormência para a germinação de sementes desta espécie (Bezerra et al., 2002; Salomão et al., 2003). Como a emergência de $C$. langsdorffii em casa de vegetação foi alta, e não diferiu estatisticamente entre os tratamentos com e sem superação de dormência (média de 94 e 90\%, respectivamente), é possível que as sementes do lote utilizado no experimento apresentassem baixo grau ou ausência de dormência. A intensidade da dormência primária, exibida por uma determinada espécie, não é uma característica fixa e pode apresentar variações em diversos níveis. Diversos estudos revelam a existência de variação no grau de dormência de sementes entre populações, entre indivíduos ou até mesmo em uma única matriz e entre diferentes anos (Andersson \& Milberg, 1998; Schütz \& Rave, 2003).

$\mathrm{O}$ estabelecimento no campo variou entre as espécies e ao longo do tempo (Figura 2). A interação entre o tratamento de superação de dormência e o tempo foi significativa para $M$. caesalpiniifolia $(p=0,007)$,
P. nitens $(\mathrm{p}=0,002)$, D. mollis $(\mathrm{p}=0,026)$ e H. stigonocarpa $(\mathrm{p}=0,001)$. Em 127 dias após a semeadura, quando a maior parte das plântulas já havia emergido, o tratamento com superação de dormência aumentou o recrutamento de quatro das seis espécies avaliadas, com exceção apenas de $P$. dubium e $C$. langsdorffii (Figura 2). Após este período, a sobrevivência das plantas diminuiu gradativamente, embora, após 320 dias da semeadura, ainda fossem detectados mais indivíduos de $M$. caesalpiniifolia e de $H$. stigonocarpa, cujas sementes tiveram a dormência superada. Ao final do experimento, a influência da superação da dormência não foi significativa para a maioria das espécies, com exceção de H. stigonocarpa (Figura 2). Até o período de 127 dias, as gramíneas, que foram removidas na implantação do experimento, já haviam crescido novamente no local, o que deve ter contribuído para aumentar a mortalidade de plantas jovens, especialmente nas espécies com sementes menores (M. caesalpiniifolia, $P$. dubium, e $P$. nitens). Pereira et al. (2013) relatam que, para estas mesmas seis espécies, a probabilidade de estabelecimento de plantas das espécies com sementes menores é maior quando as gramíneas são periodicamente removidas da área experimental, do que quando o manejo das gramíneas não é realizado. Assim, espécies de sementes menores, como $M$. caesalpiniifolia $P$. dubium e $P$. nitens, que tiveram maior probabilidade de emergência em campo, favorecida pela superação de dormência, poderiam ter apresentado maior número de indivíduos recrutados ao final do experimento, caso houvesse o manejo de gramíneas durante as primeiras fases do desenvolvimento dos indivíduos.

Tabela 2. Resultados das análises de tempo de evento, na comparação do tempo de emergência de plântulas originadas de sementes do tratamento com superação de dormência, em comparação às plântulas originadas de sementes sem superação de dormência.

\begin{tabular}{|c|c|c|c|c|}
\hline \multirow[t]{2}{*}{ Espécie } & \multicolumn{2}{|c|}{ Casa de vegetação } & \multicolumn{2}{|c|}{ Campo } \\
\hline & Estimativa \pm erro-padrão & $\chi^{2}$ & Estimativa \pm erro-padrão & $\chi^{2}$ \\
\hline Mimosa caesalpiniifolia & $-2,02 \pm 0,13$ & $261,72 * * *$ & $-1,97 \pm 0,19$ & $105,84 * * *$ \\
\hline Peltophorum dubium & $-0,54 \pm 0,13$ & $16,30 * * *$ & $-0,66 \pm 0,14$ & $21,95 * * *$ \\
\hline Pterogyne nitens & $-1,12 \pm 0,21$ & $27,36^{* * *}$ & $-1,47 \pm 0,22$ & $43,34 * * *$ \\
\hline Dimorphandra mollis & $-1,47 \pm 0,11$ & $168,62^{* * *}$ & $-2,99 \pm 0,21$ & $194,61 * * *$ \\
\hline Copaifera langsdorffii & $-0,18 \pm 0,04$ & $24,24 * * *$ & $-0,53 \pm 0,12$ & $18,51 * * *$ \\
\hline Hymenaea stigonocarpa & $-1,92 \pm 0,09$ & $451,09 * * *$ & $-1,42 \pm 0,11$ & $175,67 * * *$ \\
\hline
\end{tabular}

${ }^{n}$ Não significativo. ${ }^{*}{ }^{* *} e^{* * *}$ Significativo, pela regressão logística, a 5,1 e $0,1 \%$ de probabilidade, respectivamente. 

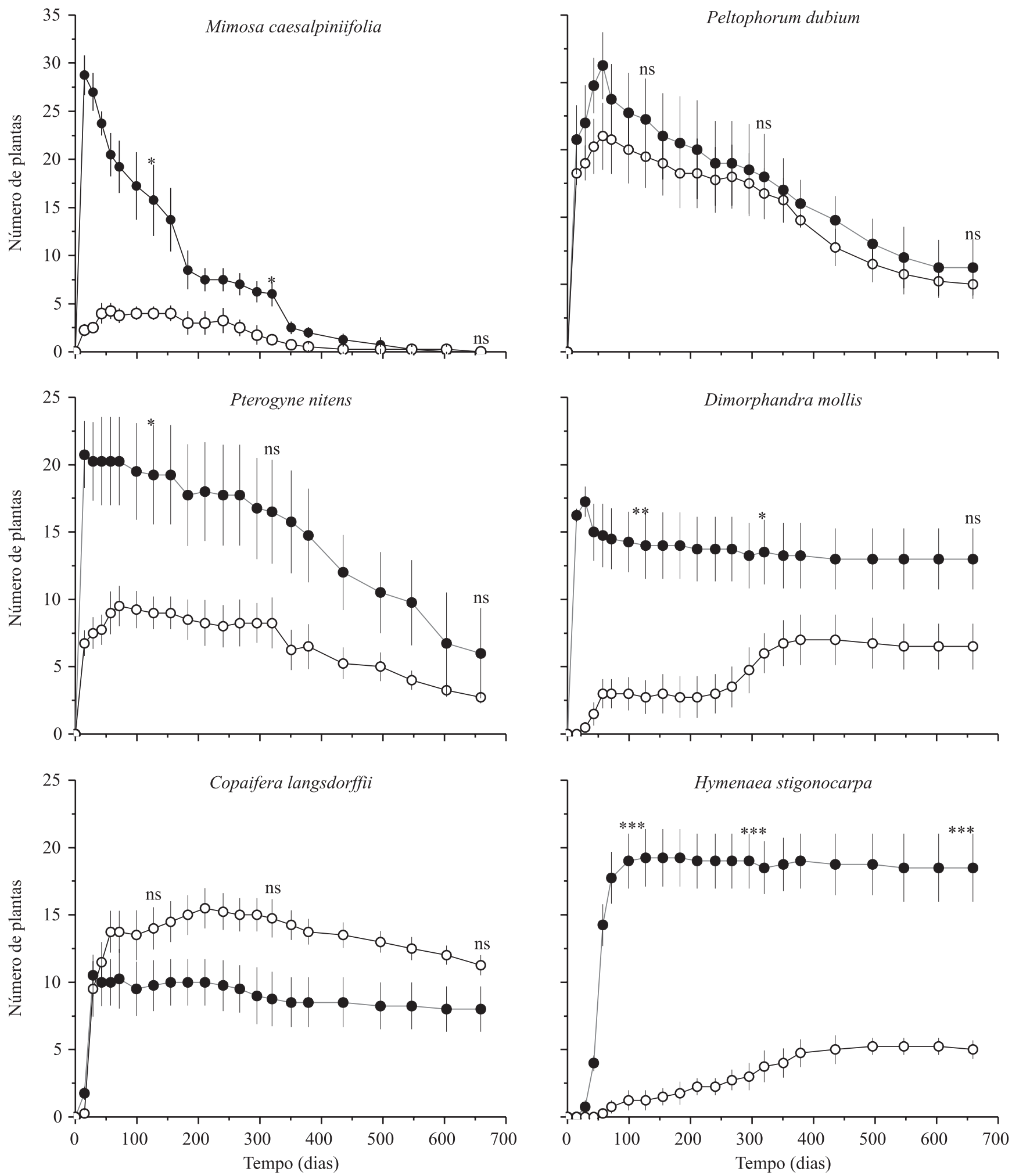

Figura 2. Recrutamento das espécies avaliadas, em tratamentos com superação de dormência (símbolos cheios) e sem superação de dormência (símbolos vazios), em área de pastagem. Barras representam o erro-padrão. ns Não significativo. ***e***Significativo, pela análise de variância de medidas repetidas, a 5, 1 e $0,1 \%$ de probabilidade, respectivamente. 


\section{Conclusões}

1. A superação de dormência de sementes aumenta a taxa de emergência da maioria das espécies arbóreas avaliadas e, consequentemente, a eficiência da técnica de semeadura direta na restauração de pastagens degradadas no Cerrado.

2. A superação de dormência diminui o tempo de emergência em campo das espécies avaliadas.

3. Asuperação de dormência aumenta o recrutamento de plantas de M. caesalpiniifolia, P. dubium, D. mollis, $C$. langsdorffii e $H$. stigonocarpa nos primeiros quatro meses após a semeadura.

\section{Agradecimentos}

À Fundação de Apoio ao Desenvolvimento do Ensino, Ciência e Tecnologia do Estado de Mato Grosso do Sul (Fundect), por apoio financeiro; e à Coordenação de Aperfeiçoamento de Pessoal de Nível Superior (Capes), por concessão de bolsa.

\section{Referências}

ANDERSSON, L.; MILBERG, P. Variation in seed dormancy among mother plants, populations and years of seed collection. Seed Science Research, v.8, p.29-38, 1998. DOI: 10.1017/ S0960258500003883.

ANDRADE, A.C.S. de; LOUREIRO, M.B.; SOUZA, A.D. de O.; RAMOS, F.N. Quebra de dormência em sementes de sucupira-preta. Pesquisa Agropecuária Brasileira, v.32, p.465-469, 1997.

BEZERRA, A.M.E.; MEDEIROS-FILHO, S.; MOREIRA, M.G; MOREIRA, F.J.C.; ALVES, T.T.L. Germinação e desenvolvimento de plântulas de copaíba em função do tamanho e da imersão da semente em ácido sulfúrico. Revista Ciência Agronômica, v.33, p.5-10, 2002.

BONILLA-MOHENO, M.; HOLL, K.D. Direct seeding to restore tropical mature-forest species in areas of slash-and-burn agriculture. Restoration Ecology, v.18, p.438-445, 2010. DOI: 10.1111/j.1526-100X.2009.00580.x.

CAMARGO, J.L.C.; FERRAZ, I.D.K.; IMAKAWA, A.M. Rehabilitation of degraded areas of central Amazonia using direct sowing of forest tree seeds. Restoration Ecology, v.10, p.636-644, 2002. DOI: 10.1046/j.1526-100X.2002.01044.x.

CHEUNG, K.C.; LIEBSCH, D.; MARQUES, M.C.M. Forest recovery in newly abandoned pastures in Southern Brazil: implications for the Atlantic Rain Forest resilience. Natureza e Conservação, v.8, p.66-70, 2010. DOI: 10.4322/natcon.00801010.

DALLING, J.W.; DAVIS, A.S.; SCHUTTE, B.J.; ARNOLD, A.E. Seed survival in soil: interacting effects of predation, dormancy and the soil microbial community. Journal of Ecology, v.99, p.89-95, 2011. DOI: 10.1111/j.1365-2745.2010.01739.x.
DOUST, S.J.; ERSKINE, P.D.; LAMB, D. Restoring rainforest species by direct seeding: tree seedling establishment and growth performance on degraded land in the wet tropics of Australia. Forest Ecology and Management, v.256, p.1178-1188, 2008. DOI: 10.1016/j.foreco.2008.06.019.

FINCH-SAVAGE，W.E.; LEUBNER-METZGER， G. Seed dormancy and the control of germination. New Phytologist, v.171, p.501-523, 2006. DOI: 10.1111/j.1469-8137.2006.01787.x.

FLORENTINE, S.K.; WESTBROOKE, M.E. Restoration on abandoned tropical pasturelands - do we know enough? Journal of Nature Conservation, v.12, p.85-94, 2004. DOI: 10.1016/j. jnc.2003.08.003.

FOX, G.A. Failure-time analysis: studying times to events and rates at which events occur. In: SCHEINER, S.M.; GUREVITCH, J. (Ed.). Design and analysis of ecological experiments. Oxford: Oxford University, 2001. p.235-266.

FRANCO, A.A.; CAMPELLO, E.F.; SILVA, E.M.R. da; FARIA, S.M. de. Revegetação de solos degradados. Seropédica: Embrapa-CNPAB, 1992. 9p. (Embrapa-CNPAB. Comunicado técnico, 9).

JACCARD, J. Interaction effects in logistic regression. London: Sage University Paper, 2001. 73p.

MARTINS, R.; CARVALHO, N.M. de; OLIVEIRA,A.P. de. Quebra de dormência de sementes de sabiá (Mimosa caesalpiniaefolia Benth.). Revista Brasileira de Sementes, v.14, p.5-8, 1992.

MATTEI, V.L.; ROSENTHAL, M.D. Semeadura direta de canafístula (Peltophorum dubium (Spreng.) Taub. No enriquecimento de capoeiras. Revista Árvore, v.26, p.649-654, 2002. DOI: 10.1590/S0100-67622002000600001.

MCIVOR, J.; HOWDEN, S.M. Dormancy and germination characteristics of herbaceous species in the seasonally dry tropics of northern Australia. Austral Ecology, v.25, p.213-222, 2000. DOI: 10.1046/j.1442-9993.2000.01026.x.

MELOTTO, A.M. Efeito do sombreamento natural no crescimento inicial de duas espécies arbóreas nativas em um sistema silvipastoril. 2008. 37p. Dissertação (Mestrado) Universidade Federal de Mato Grosso do Sul, Campo Grande.

NASSIF, S.M.L.; PEREZ, S.C.J.G. de A. Germinação de sementes de amendoim-do-campo (Pterogyne nitens Tul.): influência dos tratamentos para superar a dormência e profundidade de semeadura. Revista Brasileira de Sementes, v.19, p.171-178, 1997.

NEPSTAD, D.C.; UHL, C.; PEREIRA, C.A.; SILVA, J.M.C. da. A comparative study of tree establishment in abandoned pasture and mature forest of astern Amazonia. Oikos, v.76, p.25-39, 1996. DOI: $10.2307 / 3545745$.

OLIVEIRA, L.M. de; DAVIDE, A.C.; CARVALHO, M.L.M. de. Teste de germinação de sementes de Peltophorum dubium (Sprengel) Taubert - Fabaceae. Floresta, v.38, p.545-551, 2008.

PEREIRA, S.R.; GIRALDELLI, G.R.; LAURA, V.A.; SOUZA, A.L.T. de. Tamanho de frutos e de sementes e sua influência na germinação de jatobá-do-cerrado (Hymenaea stigonocarpa var. stigonocarpa Mart. ex Hayne, Leguminosae - Caesalpinoideae). Revista Brasileira de Sementes, v.33, p.141-148, 2011. DOI: 10.1590/S0101-31222011000100016. 
PEREIRA, S.R.; LAURA, V.A.; SOUZA, A.L.T. Establishment of Fabaceae tree species in a tropical pasture: influence of seed size and weeding methods. Restoration Ecology, v.21, p.67-74, 2013. DOI: 10.1111/j.1526-100X.2011.00858.x.

REGRAS para análise de sementes. Brasília: Ministério da Agricultura, Pecuária e Abastecimento, 2009. 395p.

SALOMÃO N.A.; SOUZA-SILVA, J.C.; DAVIDE, A.C.; GONZÁLES, S.; TORRES, R.A.A.; WETZEL, M.M.V.S.; FIRELI, F.; CALDAS, L.S. Germinação de sementes e produção de mudas de plantas do Cerrado. Brasília: Rede de Sementes do Cerrado, 2003. 96p.

SCHMIDT, L. A review of direct sowing versus planting in tropical afforestation and land rehabilitation. Copenhagen: Forest and Landscape, 2008. 37p. (Development and environment, 10).

SCHÜTZ, W.; RAVE, G. Variation in seed dormancy of the wetland sedge, Carex elongata, between populations and individuals in two consecutive years. Seed Science Research, v.13, p.315-322, 2003. DOI: $10.1079 /$ SSR2003148.
SIDDIQUE, I.; ENGEL, V.L.; PARROTTA, J.A.; LAMB, D.; NARDOTO, G.B.; OMETTO, J.P.H.B.; MARTINELLI, L.A.; SCHMIDT, S. Dominance of legume trees alters nutrient relations in mixed species forest restoration plantings within seven years. Biogeochemistry, v.88, p.89-101, 2008. DOI: 10.1007/ s10533-008-9196-5.

SOVU, P.S.; TIGABU, M.; ODÉN, P.C. Restoration of former grazing lands in the highlands of Laos using direct seeding of four native tree species. Mountain Research and Development, v.30, p.232-243, 2010. DOI: 10.1659/ MRD-JOURNAL-D-10-00031.1.

VIGLIZZO, E.F.; FRANK, F.C. Land-use options for Del Plata Basin in South America: tradeoffs analysis based on ecosystem service provision. Ecological Economics, v.57, p.140-151, 2006. DOI: 10.1016/j.ecolecon.2005.03.025.

WOODS, K.; ELLIOTT, S. Direct seeding for forest restoration on abandoned agricultural land in northern Thailand. Journal of Tropical Forest Science, v.16, p.248-259, 2004.

Recebido em 10 de agosto de 2012 e aprovado em 30 de janeiro de 2013 